\title{
Metric properties in the mean of polynomials on compact isotropy irreducible homogeneous spaces
}

\author{
V.M. Gichev*
}

\begin{abstract}
Let $M=G / H$ be a compact connected isotropy irreducible Riemannian homogeneous manifold, where $G$ is a compact Lie group (may be, disconnected) acting on $M$ by isometries. This class includes all compact irreducible Riemannian symmetric spaces and, for example, the tori $\mathbb{R}^{n} / \mathbb{Z}^{n}$ with the natural action on itself extended by the finite group generated by all permutations of the coordinates and inversions in circle factors. We say that $u$ is a polynomial on $M$ if it belongs to some $G$-invariant finite dimensional subspace $\mathcal{E}$ of $L^{2}(M)$. We compute or estimate from above the averages over the unit sphere $\mathcal{S}$ in $\mathcal{E}$ for some metric quantities such as Hausdorff measures of level set and norms in $L^{p}(M), 1 \leq p \leq \infty$, where $M$ is equipped with the invariant probability measure. For example, the averages over $\mathcal{S}$ of $\|u\|_{L^{p}(M)}, p \geq 2$, are less than $\sqrt{\frac{p+1}{e}}$ independently of $M$ and $\mathcal{E}$.
\end{abstract}

\section{Introduction}

Let $M$ be a compact connected Riemannian manifold, $G$ be a compact Lie group acting on $M$ transitively by isometries, and $H$ be the stable subgroup of a base point $o \in M$. We assume that $M$ is isotropy irreducible, i.e., that the group $H$ has no proper invariant subspaces in $T_{o} M$ (the action of $H$ is induced by its adjoint representation in the Lie algebra $\mathfrak{g}$ of $G$ ). This class of homogeneous spaces is rather wide - it includes all irreducible Riemannian symmetric spaces, in particular, real spheres, Grassman manifolds, and simple Lie groups. The mentioned spaces are strongly isotropy irreducible, i.e., the connected component of $H$ is irreducible in $T_{o} M$. A torus $T$ considered as a homogeneous space of the semidirect product of $T$ and a finite group $F$ of its isometrical automorphisms is not strongly isotropy irreducible but it can be isotropy irreducible (this happens if and only if $F$ is irreducible in $T_{o} M$ ). The circle group $\mathbb{T}=\mathbb{R} / 2 \pi \mathbb{Z}$ is also contained in this class.

${ }^{*}$ Part of the work was done during my stay in the Institut Mittag-Leffler (Djursholm, Sweden), 2011 fall. I thank the Institut for support and hospitality. 
We say that a function $u$ on $M$ is a polynomial if the linear span of its translates $u \circ g, g \in G$, is finite dimensional. The polynomials are real analytic functions on $M$ since they can be lifted onto $G$ as matrix elements of finite dimensional representations. Let $\mathcal{E}$ be a finite dimensional $G$-invariant linear subspace of $L^{2}(M)$ and $\mathcal{S}$ be the unit sphere in it. (In the notation $L^{2}(M)$, the probability invariant measure on $M$ is assumed.) In this paper, we compute or estimate the averages over $\mathcal{S}$ of some metric quantities, such as the Hausdorff measures of level sets and their intersections or $L^{p}$-norms of polynomials $u \in \mathcal{S}$.

The strongly isotropy irreducible homogeneous spaces were classified first by O.V. Manturov ([21]-[23]) in 1961, independently by J.A. Wolf ([41]) in 1968, and by M. Krämer ([17]) in 1975. Their structure was clarified in papers by M. Wang and W. Ziller (38]), E. Heintze and W. Ziller ([13]). This class of homogeneous spaces is closely connected with the symmetric spaces.

Due to Schur's lemma, $M$ admits a unique up to a scaling factor $G$-invariant Riemannian metric. It follows from the uniqueness that it is a quotient of some bi-invariant metric on $G$. We fix these metrics and denote by $\Delta$ and $\tilde{\Delta}$ the corresponding Laplace-Beltrami operators on $M$ and $G$, respectively.

Let the Lie algebra $\mathfrak{g}$ of $G$ be realized by right invariant vector fields on $G$. The Lie algebra of their projections onto $M$ is its homomorphic image; if the action is virtually effective 1 , then the projection is an isomorphism. We shall assume this. Thus, we may identify these Lie algebras. If $\xi_{1}, \ldots, \xi_{l}$ is an orthonormal base in $\mathfrak{g}$, then $\tilde{\Delta}=\xi_{1}^{2}+\cdots+\xi_{l}^{2}$, where $l=\operatorname{dim} G$, and

$$
\Delta=\xi_{1}^{2}+\cdots+\xi_{l}^{2},
$$

where $\xi_{j}$ denotes a vector field on $G$ as well as its projection onto $M$. Note that $\xi_{j}$ may vanish on $M: \xi_{j}(p)=0$ if and only if $\xi \in \mathfrak{h}_{p}$, the stable subalgebra for $p \in M$. Since the vector fields in $\mathfrak{g}$ generate one parameter subgroups of $G$, we have $\xi \mathcal{E} \subseteq \mathcal{E}$ for any $G$-invariant finite dimensional linear subspace $\mathcal{E}$ of $L^{2}(M)$ and all $\xi \in \mathfrak{g}$. By (1),

$$
\Delta \mathcal{E} \subseteq \mathcal{E}
$$

If $M$ is not isotropy irreducible, then (2) may be false. (It is true if the metric on $M$ is a quotient of a bi-invariant metric on $G$.) Also, it follows from (2) that any polynomial on $M$ is a finite linear combination of eigenfunctions of $\Delta$.

Throughout the paper, we use the notation

$$
m=\operatorname{dim} M .
$$

For a real function $u$ on $M$ and $t \in \mathbb{R}$, set

$$
\begin{aligned}
L_{u}^{t} & =\{p \in M: u(p)=t\}, \\
U_{u}^{t} & =\{p \in M: u(p) \geq t\} .
\end{aligned}
$$

\footnotetext{
${ }^{1}$ The action is effective if its kernel is trivial and virtually effective if it is finite. The kernel of the action consists of those $g \in G$ which define the identical transformation of $M$.
} 
If $u$ is an eigenfunction of $\Delta$, then $L_{u}^{0}$ is called a nodal set; we shall also use the notation $N_{u}$ for it. The Hausdorff measure of dimension $k$ is denoted as $\mathfrak{h}^{k}$. We assume $t$ fixed and consider Hausdorff measures of these sets as functions of $u$, and, for example, $\mathfrak{h}^{m}\left(U_{u_{1}}^{t_{1}} \cap \ldots \cap U_{u_{k}}^{t_{k}}\right)$ as functions of $u_{1}, \ldots, u_{k}$. Fixing a probability measure on $\mathcal{E}$ and $t \in \mathbb{R}$, we get random variables $\mathfrak{h}^{m-1}\left(L_{u}^{t}\right), \mathfrak{h}^{m}\left(U_{u}^{t}\right)$, etc.. Their distributions contain essential information on the polynomials.

\section{$1.1 \quad$ Brief history}

To the best of my knowledge, investigations in this direction were initiated by papers [4 by Bloch and Polya, 30] by Paley, Wiener, and Zygmund, and [19], [20 by Littlewood and Offord. They considered the number of real zeroes of algebraic equations with various types of random coefficients. There are many papers in this area now; we describe briefly only the results which are close to this article.

For real zeroes of random polynomials of one variable M. Kac in 15] proved an exact integral formula for the expectation and found its asymptotic. Edelman and Kostlan in the paper [8] noted that the expectation may be treated as the length of some curve in a sphere due to a Crofton type formula in spheres. They used this approach in some other situations.

For the Laplace-Beltrami eigenfunctions on compact manifolds, Berard found the asymptotic of expectations of $\mathfrak{h}^{m-1}\left(N_{u}\right)$, where $u$ runs over the linear span of eigenfunctions corresponding to the eigenvalues of $-\Delta$ which are less than $\lambda$, as $\lambda \rightarrow \infty$ (see [3] for more details).

The case of $\mathbb{T}$ is classical. We mention only papers [31, [5], [12. For the trigonometric polynomials

$$
u=\frac{1}{\sqrt{n}} \sum_{k=1}^{n}\left(a_{k} \cos k t+b_{k} \sin k t\right)
$$

where $a_{k}, b_{k}$ are Gaussian standard (i.e., with zero mean and variance 1 ) random coefficients, Qualls (31]) found the expectations $E_{n}$ of the number of zeroes $Z_{n}(u)=\mathfrak{h}^{0}\left(N_{u}\right)$ :

$$
E_{n}=2 \sqrt{\frac{1}{n} \sum_{k=1}^{n} k^{2}} \sim \frac{2}{\sqrt{3}} n .
$$

Bogomolny, Bohigas and Leboeuf conjectured in [5 that var $Z_{n}=c n$ for some $c>0$. Granville and Wigman proved this equality and, moreover, that $\frac{Z_{n}(u)-E_{n}}{\sqrt{c n}}$ converges weakly to the standard Gaussian distribution ([12]). The constant $c$ is equal to some complicated explicitly written definite integral.

In [28, Oravecz, Rudnick, and Wigman considered the standard tori $\mathbb{R}^{m} / \mathbb{Z}^{m}$, a suitably normalized Gaussian measure in the space $\mathcal{E}_{\lambda}$ of $\lambda$-eigenfunctions, and the Leray measure of a nodal set

$$
\mathfrak{l}\left(N_{u}\right)=\lim _{\varepsilon \rightarrow 0} \frac{1}{2 \varepsilon} \mathfrak{h}^{m}\left(U_{u}^{-\varepsilon} \backslash U_{u}^{\varepsilon}\right) .
$$


Note that the space $\mathcal{E}_{\lambda}$ of all $\lambda$-eigenfunctions on $\mathbb{R}^{m} / \mathbb{Z}^{m}$ is always invariant with respect to the permutations of the coordinates and changes of their signs, in other words, it is an invariant subspace of the semidirect product $G$ of the torus $\mathbb{R}^{n} / \mathbb{Z}^{n}$ and the finite irreducible group $\mathrm{BC}_{n}$, which is described above. (In fact, the results of [28] were obtained for $G$-invariant subspaces of $\mathcal{E}_{\lambda}$.) They calculated the expectation of $\mathfrak{l}\left(N_{u}\right)$, which appears to be equal to $\frac{1}{\sqrt{2 \pi}}$ independently of $m$ and $\lambda$, and proved for $m=2$ and $m \geq 5$ that

$$
\operatorname{varl}\left(N_{u}\right) \sim \frac{1}{4 \pi \operatorname{dim} \mathcal{E}_{\lambda}}
$$

as $\lambda \rightarrow \infty$. In 32, Rudnick and Wigman proved that the expectation of $\mathfrak{h}^{m-1}\left(N_{u}\right)$ is asymptotic to $C \sqrt{\lambda}$ and

$$
\operatorname{var}\left(\lambda^{-\frac{1}{2}} \mathfrak{h}^{m-1}\left(N_{u}\right)\right)=O\left(\lambda^{-\frac{1}{2}}\right)
$$

for the Gaussian distributions of $u \in \mathcal{E}_{\lambda}$ on the tori $\mathbb{R}^{m} / \mathbb{Z}^{m}, m \geq 2$, assuming $\operatorname{dim} \mathcal{E}_{\lambda} \rightarrow \infty$.

Let $S^{m}$ denote the unit sphere in $\mathbb{R}^{m+1}$. The $n$th eigenspace $\mathcal{E}_{\lambda_{n}}=\mathcal{H}_{n}^{m}$ corresponds to the eigenvalue $\lambda_{n}=n(n+m-1)$ and consists of traces of harmonic homogeneous of degree $n$ polynomials on $S^{m}$. This case was considered in the papers [27, 11], 39], 40], 24], 25], where $u$ was subject either to the Gaussian distribution in $\mathcal{E}_{\lambda}$ or to the uniform one in $\mathcal{S}$.

Remark 1. Both distributions mentioned above are rotation invariant. Since $\mathfrak{h}^{m-1}\left(N_{u}\right)$ and $\mathfrak{h}^{m}\left(U_{u}^{0}\right)$ are homogeneous of degree 0 on $u$, the resulting distributions of $\mathfrak{h}^{m-1}\left(N_{u}\right)$ and $\mathfrak{h}^{m}\left(U_{u}^{0}\right)$ are identical in the Gaussian and the uniform spherical cases. In particular, the expectations and variances are equal. For the level sets and for the Leray measures, this is not true but the results for any of the two types of distributions can be deduced from the results on the other one (for instance, we compute the expectations for radial measures in Proposition (1). In the papers cited above, except for the first and the second, the authors work with the Gaussian distribution.

Neuheisel proved that the normalized Hausdorff and Leray measures on the nodal sets almost surely converges $*$-weakly to the probability invariant measure as $\lambda \rightarrow \infty$ (for the precise statement, see [27]). He found the expectations of $\mathfrak{h}^{m-1}\left(N_{u}\right)$ and $\mathfrak{l}\left(N_{u}\right)$ and estimated their variances as $O\left(n^{-\frac{(m-1)^{2}}{3 m+1}}\right)$ and $O\left(n^{-\frac{m-1}{2}}\right), n \rightarrow \infty$, respectively. In [39], Wigman refined this: he proved that $\operatorname{var}\left(\mathfrak{l}\left(N_{u}\right)\right)=\frac{c}{N}$, where $c$ depends only on $m, N=\operatorname{dim} \mathcal{H}_{m}^{n}$, and $\operatorname{var}\left(\mathfrak{h}^{m-1}\left(N_{u}\right)\right)=O\left(\frac{\lambda}{\sqrt{N}}\right)$. For $S^{2}$ he proved that $\operatorname{var}\left(\mathfrak{h}^{1}\left(N_{u}\right)\right)=c \ln n+O(1)$ in [40] (there was an error in the calculation of $c$ which had been corrected later). Marinucci and Wigman studied the random area $\mathfrak{h}^{2}\left(S^{2} \backslash U_{u}^{t}\right)$. In [24, they show that for a fixed $t \in \mathbb{R}$

$$
\operatorname{var}\left(\mathfrak{h}^{2}\left(S^{2} \backslash U_{u}^{t}\right)\right)=\frac{t^{2} \phi(t)}{n}+O\left(\frac{\log n}{n^{2}}\right)
$$


as $n \rightarrow \infty$, where $\phi$ is the standard Gaussian distribution function. For $t=0$, it is proved in 25] that $\operatorname{var}\left(U_{u}^{0}\right)=\frac{C}{n^{2}}(1+o(1))$, where $n$ is even,

$$
C=8 \pi \int_{0}^{\infty}\left(\arcsin J_{0}(\tau)-J_{0}(\tau)\right) \tau d \tau,
$$

$J_{0}$ is the Bessel function of the first kind and zero index; the integral converges conditionally. (Actually, the authors considered the difference between measures of sets of positivity and negativity of $u$ (defect); their result differs by the factor 4 from $C$ above.)

In [11, estimates for some metric quantities of the nodal sets in spheres (in particular, the sharp upper bound for lengths of the nodal sets of spherical harmonics on $S^{2}$ ) and the expectations of Hausdorff measures of their intersections for the uniform distributions were found, including the mean number of common zeroes of $m$ independent random eigenfunctions on $S^{m}$.

Surveys [14, 42, 43] describe the current state of this area. They contain many known facts as well as methods and open problems.

\subsection{Some observations and the results}

In this paper, we consider an arbitrary compact connected isotropy irreducible homogeneous manifold $M$ and expectations of some metric quantities for polynomials. The results on variances, higher moments, and estimates usually cannot be proved in such generality; they will be considered in forthcoming papers. Throughout the text it is assumed that

(E) $\mathcal{E}$ is a finite dimensional $G$-invariant linear subspace of $L^{2}(M)$ such that $\mathbf{1} \perp \mathcal{E}$, where $\mathbf{1}(p)=1$ for all $p \in M$.

Also, $\mathcal{S}$ everywhere denotes the unit sphere in $\mathcal{E}$. Unless the contrary is explicitly stated, the expectations relate to the uniform distribution in $\mathcal{S}$ (i.e., to the $\mathrm{SO}(\mathcal{E})$-invariant probability measure).

We formulate below some useful observations.

1. Let $N$ be a Riemannian $G$-manifold and $\iota: M \rightarrow N$ be an equivariant nonconstant smooth map. Then $\iota$ is a local diffeomorphism onto its image since $M$ is isotropy irreducible (hence $\operatorname{ker} d_{p} \iota=0$ for all $p \in M$ ). Therefore, $\iota$ is a finite covering.

2. The restriction of the Riemannian metric in $N$ onto $\iota(M)$ is proportional to the Riemannian metric in $M$ since the invariant Riemannian metric in $M$ is unique up to a scaling factor.

3. Let $s$ be the coefficient of proportionality. If $\gamma$ is a path in $M$ of length $l$, then the path $\iota \circ \gamma$ has length $s l$. It follows that the inner distances locally are also multiplied by $s$ (i.e., $\iota$ is a loca 2 metric homothety) and the same is true for the Hausdorff measure $\mathfrak{h}^{k}$, with the coefficient $s^{k}$.

\footnotetext{
${ }^{2}$ The two-sheeted expending covering $z \rightarrow z^{2}$ of the unit circle $\mathbb{T}$ in $\mathbb{C}$ is a simple example of a non-global local homothety
} 
4. There is a natural equivariant immersion $\iota: M \rightarrow \mathcal{S}$. For $p \in M$, let $\phi_{p} \in \mathcal{E}$ be such that $u(p)=\left\langle\phi_{p}, u\right\rangle$ for all $u \in \mathcal{E}$ and set $\iota(p)=\frac{\phi_{p}}{\left|\phi_{p}\right|}$.

5. For the immersion $\iota$ we have $s=\sqrt{\frac{|\operatorname{Tr} \Delta|}{\operatorname{dim} M \operatorname{dim} \mathcal{E}}}$ (see Lemma 1 below). If $\mathcal{E}$ is an eigenspace of $\Delta$, then $s=\sqrt{\frac{\lambda}{m}}$, where $\lambda$ is the eigenvalue of $-\Delta$.

6. Using a Crofton type formula of Integral Geometry in spheres (see Theorem 11), one can compute averages (expectations) of $\mathfrak{h}^{k-1}\left(L_{u}^{t} \cap X\right)$ ), $\left.\mathfrak{h}^{k}\left(U_{u}^{t} \cap X\right)\right)$ for subsets $X$ of $M$, and some other functions of $u$, with respect to the probability invariant measure on the sphere $\mathcal{S}$.

They seem to be already known (may be, except for the third and the fifth; the coefficient $s$ appeared implicitly in several papers, for example, in [27]). The scheme was realized in the paper [11] for $M=S^{m}=\mathrm{SO}(m+1) / \mathrm{SO}(m)$, the unit sphere in $\mathbb{R}^{m+1}$ and the spaces of spherical harmonics on them, which can be characterized as eigenspaces of $\Delta$ or as irreducible components of $L^{2}\left(S^{m}\right)$. Clearly, $S^{m}$ is isotropy irreducible, moreover, the stable subgroup $H=\mathrm{SO}(m)$ acts transitively on the unit sphere in $T_{o} S^{m}$. It turns out that the assumption that $\mathcal{E}$ is an eigenspace of $\Delta$ is not essential for the computation of expectations, the results depends (at most) on the coefficient $s$. (However, for variances and upper or lower bounds this is not true usually.)

We compute the expectations of Hausdorff measures of level sets and their intersections (Theorem 2). In particular, for $L_{u}^{t}$ and $U_{u}^{t}$ we get

$$
\begin{gathered}
\mathrm{M}\left(\mathfrak{h}^{m-1}\left(L_{u}^{c t}\right)\right)=\varpi \frac{\varpi_{m-1}}{\varpi_{m}} s\left(1-t^{2}\right)^{\frac{d-1}{2}}, \\
\mathrm{M}\left(\mathfrak{h}^{m}\left(U_{u}^{c t}\right)\right)=\varpi \frac{\varpi_{d-1}}{\varpi_{d}} \int_{t}^{1}\left(1-\tau^{2}\right)^{\frac{d}{2}-1} d \tau,
\end{gathered}
$$

where $m=\operatorname{dim} M, \mathcal{E}, s$ are as above, $c^{2}=d+1=\operatorname{dim} \mathcal{E}, \varpi, \varpi_{k}$ are volumes of $M, S^{k}$, respectively, $t \in \mathbb{R}$ is fixed, and

$$
\mathrm{M}(f)=\int_{\mathcal{S}} f(u) d u
$$

for a function $f$ on $\mathcal{S}$ ( $d u$ corresponds to the invariant probability measure on $\mathcal{S})$.

The formulas above hold for all isotropy irreducible homogeneous spaces, in particular, for spheres $\mathrm{SO}(m+1) / \mathrm{SO}(m)$ and for the standard tori $T_{m}=$ $\mathbb{R}^{m} / \mathbb{Z}^{m}$ considered as a homogeneous space of $T_{m}$ extended by the finite group $\mathrm{BC}_{m}$ of all compositions of permutations and componentwise inversions in $T_{m}$. This is equivalent to the assumption that the $T_{m}$-invariant space $\mathcal{E}$ (equivalently, its spectrum) is $\mathrm{BC}_{m}$-invariant; in fact, it was assumed in the paper 28] (clearly, the spectrum of any eigenspace on $T_{m}$ is $\mathrm{BC}_{m}$-invariant). Qualls's formula (4) follows from (6) with $M=G=\mathbb{T}=\mathbb{R} / 2 \pi \mathbb{Z}$ and $t=0$ since $\varpi_{0}=2$, $\varpi=\varpi_{1}=2 \pi$, and $s=\sqrt{\frac{1}{n} \sum_{k=1}^{n} k^{2}}$ according to Lemma 11. Moreover, (6) 
can be applied to spaces $\mathcal{E}$ with arbitrary spectra, for example, to the space of trigonometric polynomials of the type

$$
\sum_{i=1}^{n}\left(a_{k_{i}} \cos k_{i} t+b_{k_{i}} \sin k_{i} t\right)
$$

where $0<k_{1}<\cdots<k_{n}$ are integer. By (6) and Lemma 1, the expectation equals $2 s$, where

$$
s=\sqrt{\frac{1}{n} \sum_{i=1}^{n} k_{i}^{2}}
$$

A similar formula can be derived for the intersections of sets $L_{u}^{t}$ and $U_{u}^{t}$, for the natural extension of the Leray measure onto all level sets (see (16) for the definition), and for quantities of the type $\int_{M} f(u(p)) d p$. The results are stated in Theorem 2 For $f(t)=|t|^{a}$ we derive the explicit formula (47) (Theorem 3), which holds for all $a>-1$; its right-hand side is independent of $M$. Setting $a=1$, we get the expectation of $L^{1}$-norm:

$$
\mathrm{M}\left(\|u\|_{1}\right)=\sqrt{\frac{d+1}{\pi}} \frac{\Gamma\left(\frac{d+1}{2}\right)}{\Gamma\left(\frac{d+2}{2}\right)}
$$

where $d=\operatorname{dim} \mathcal{S}=\operatorname{dim} \mathcal{E}-1$. The right-hand side decreases with $d$. If $d=1$, then it is equal to $\frac{2 \sqrt{2}}{\pi} \approx 0.9$ and it tends to $\sqrt{\frac{2}{\pi}} \approx 0.8$ as $d \rightarrow \infty$. For any $a>-1$ we have

$$
E(a, d):=\mathrm{M}\left(\int_{M}|u(p)|^{a} d p\right) \rightarrow 2^{\frac{a}{2}} \frac{\Gamma\left(\frac{a+1}{2}\right)}{\sqrt{\pi}},
$$

as $d \rightarrow \infty$, where $E(a, d)$ increases with $d$ if $a>2$ or $a \in(-1,0)$ and decreases if $a \in(0,2)$ (for $a=0$ and $a=2$ the equality holds). As $a \rightarrow-1, E(a, d) \sim \frac{A}{a+1}$, where $A$ depends only on $d$. The integral $\int_{M}|u(p)|^{a} d p$ may diverge for arbitrary small negative $a$, for example, if $M=\mathbb{T}$ and $u(t)=(\sin t)^{2 k+1}$, but the averages are finite if $a>-1$.

The computation of $E(a, d)$ makes it possible to estimate from above the expectation of norms $\|u\|_{p}$ in $L^{p}(M), 1 \leq p<\infty$ (Theorem 4). If $p \geq 2$, then

$$
\mathrm{M}\left(\|u\|_{p}\right)<\sqrt{\frac{p+1}{e}} .
$$

For $p \in[1,2)$ the same inequality holds if $\operatorname{dim} \mathcal{E}$ is sufficiently large. Note that the bound is independent of $\mathcal{E}$ and $M$. Inequalities like $\|u\|_{p} \leq C \sqrt{p}\|u\|_{2}$ are known for the trigonometric lacunary series. Perhaps, this upper bound cannot be improved essentially.

The uniform estimate for the expectations does not yield a similar estimate for individual eigenfunctions. For example, if $M=S^{2} \subset \mathbb{R}^{3}$, then for any $p>2$ and spherical harmonics $\varphi_{n}(x, y, z)=c \operatorname{Re}(x+y i)^{n}$ of degree $n$ such that 
$\left\|\varphi_{n}\right\|_{2}=1$ we have $\left\|\varphi_{n}\right\|_{p} \rightarrow \infty$ as $n \rightarrow \infty$ (see [36]). On the other hand, if $M=\mathbb{R}^{2} / \mathbb{Z}^{2}$, then $\|u\|_{4} \leq C\|u\|_{2}$ for all eigenfunctions $u$ by a result of Zygmund (44]). Similar problems for $L^{4}$ norms of elements of a random orthogonal base in the space $\mathcal{H}_{n}$ of spherical harmonics on $S^{2}$ of degree $n$ were considered in [37; in particular, this paper contains a sketch of the proof of an estimate for the average of the functional $\sum_{j=1}^{2 n+1}\left\|u_{k}\right\|_{4}^{4}$ of such a base.

Estimates for $\mathrm{M}\left(\|u\|_{\infty}\right)$ cannot be derived from the results on $\mathrm{M}\left(\|u\|_{p}\right)$ directly while for any fixed $u \in \mathcal{E}$ we have $\|u\|_{p} \rightarrow\|u\|_{\infty}$ as $p \rightarrow \infty$. Indeed, the upper bound (8) for $\mathrm{M}\left(\|u\|_{p}\right)$ is independent of $\operatorname{dim} \mathcal{E}$ but it may happen that $\mathrm{M}\left(\|u\|_{\infty}\right) \rightarrow \infty$ as $\operatorname{dim} \mathcal{E} \rightarrow \infty$, for example, this is true if $\mathcal{E}$ is contained in a subspace of $L^{2}(\mathbb{T})$ with a lacunary spectrum. There is the evident sharp upper bound $\sqrt{\operatorname{dim} \mathcal{E}}=: c$ for $\|u\|_{\infty}$ (see (12)) which is attained on $u=\iota(q) \in \mathcal{S}$ for any $q \in M$. We get the estimate

$$
\begin{array}{r}
\mathrm{M}\left(\|u\|_{\infty}\right)<\left(e^{m-\frac{1}{2}}+\varepsilon\right) \sqrt{\ln \kappa}, \\
\kappa=c s,
\end{array}
$$

which holds for any $\varepsilon>0$ and sufficiently large $\kappa$. (In fact, $\kappa$ is the norm of the identity operator $\mathcal{E} \rightarrow \mathcal{E}$ with norms of $L^{2}(M)$ and $\operatorname{Lip}(M)$, respectively; see Lemma 5.) Similar upper bounds for the random variable $\|u\|_{\infty}$ appeared in various problems. For instance, the analogous inequalities of weak type for $\|u\|_{\infty}$ are contained in Kahane's book [16, Ch. 6]; in [27, Neuheisel proved for spherical harmonics on $S^{m}$ that $\left\|u_{n}\right\|_{\infty}=O(\sqrt{\ln n})$ almost surely as $n \rightarrow \infty$, where $u_{n} \in \mathcal{S}_{n} \subseteq \mathcal{E}_{n}$ and $\mathcal{E}_{n}$ is the space of spherical harmonics of degree $n$. In these cases, the bounds $\sqrt{\ln \kappa}, \sqrt{\ln s}, \sqrt{\ln c}$, and $\sqrt{\ln n}$ are equivalent. Shiffman and Zelditch in the paper [34] considered spaces $\mathcal{H}_{n}=H\left(M, \mathcal{L}^{n}\right)$ of holomorphic sections of the $n$th power of a positive line bundle $\mathcal{L}$ over a compact Kähler manifold $M$. These spaces are treated as subspaces of $L^{2}(X)$, where $X$ is the $S^{1}$ bundle associated to $\mathcal{L}$. (If $M$ is the projective space $\mathbb{C P}^{m}$ and $\mathcal{L}$ is the natural line bundle over it, then $\mathcal{H}_{n}$ is the space of holomorphic homogeneous polynomials of degree $n$ on $\mathbb{C}^{m+1}$ restricted to the unit sphere $X=$ $S^{2 m+1}$.) Such a construction can be applied to the almost complex manifolds. In particular, they proved that $L^{p}$-norms of sequences of elements of the unit spheres $\mathcal{S H}_{n}$ in $\mathcal{H}_{n}$ are $O(1)$ and $O(\sqrt{\log n})$ almost surely as $n \rightarrow \infty$ for $p<\infty$ and $p=\infty$, respectively. The key ingredients of their methods, which can work in the real setting as well, are the asymptotics of reproducing kernels and the concentration of measure estimates (see, e.g., [18]). The proof of (9) in this paper is based on Lemma 5 and the estimate (8).

The inequality (9) contains no information if $K^{2} \ln \kappa>\operatorname{dim} \mathcal{E}$. This happens, for example, for the spaces of trigonometric polynomials with the spectrum $\{1,2, \ldots, n, n$ ! $\}$ if $n$ is sufficiently large. Thus, (9) should be refined. Probably, the right-hand side of (9) exhibits the sharp order of growth for strictly isotropy irreducible homogeneous spaces if $\mathcal{E}$ is an eigenspace. 


\section{Preparatory material}

In what follows, we keep the notation of the introduction; || and $\langle$,$\rangle denote$ the norm and inner product in Euclidean spaces, respectively, \|\|$_{p}$ is the norm in $L^{p}(M)$. We write $\int_{M} f(p) d p, \int_{G} f(g) d g$, etc., for the integration over invariant probability measures on $M, G$, and other homogeneous spaces of compact groups; also, these measures are assumed in the notation $L^{2}(M), L^{2}(G)$, etc.. Thus, we consider on $M$ two finite invariant measures: the probability one and $\mathfrak{h}^{m}$, where $m=\operatorname{dim} M$. Set

$$
\varpi=\mathfrak{h}^{m}(M) ;
$$

then $d \mathfrak{h}^{m}=\varpi d p$. Functions are assumed to be real-valued unless the contrary is explicitly stated. The space $\mathcal{E}$ is always assumed to satisfy (E). Since we consider real functions and $M$ is compact, (E) implies

$$
\operatorname{dim} \mathcal{E}>1 .
$$

Being finite dimensional and $G$-invariant, $\mathcal{E}$ consists of real analytic functions. For any $p \in M$ there exists the unique $\phi_{p} \in \mathcal{E}$ which realizes the evaluation functional at $p$ :

$$
\left\langle u, \phi_{p}\right\rangle=u(p)
$$

for all $u \in \mathcal{E}$. Set

$$
\phi(p, q)=\left\langle\phi_{p}, \phi_{q}\right\rangle, \quad p, q \in M .
$$

Then $\phi(p, q)=\phi_{p}(q)=\phi(q, p)$; moreover, $\phi(x, y)$ is the reproducing kernel for $\mathcal{E}$ (i.e., the mapping $u(x) \rightarrow \int_{M} \phi(x, y) u(y) d y$ is the orthogonal projection onto $\mathcal{E}$ in $\left.L^{2}(M)\right)$. Due to the homogeneity of $M,\left|\phi_{p}\right|=\left|\phi_{o}\right| \neq 0$ for all $p \in M$. Since the trace of the projection is equal to $\int_{M} \phi(x, x) d x$, we have

$$
\phi(o, o)=\left|\phi_{o}\right|^{2}=\operatorname{dim} \mathcal{E} .
$$

By the first observation on Page 5 , the equivariant mapping $\iota: M \rightarrow \mathcal{S}$,

$$
\iota(p)=\frac{\phi_{p}}{\left|\phi_{p}\right|}
$$

of the forth observation is an immersion. The denominator in (10) is independent of $p$ since $M$ is homogeneous. Clearly, $\iota$ is an embedding if and only if $\mathcal{E}$ separates points of $M$.

The scaling factor $s$ of the second observation admits an evident expression on the level of tangent spaces:

$$
s=s(\mathcal{E})=\frac{\left|d_{p} \iota(v)\right|}{|v|}
$$


where the right-hand side is independent of $p \in M$ and $v \in T_{p} M \backslash\{0\}$. According to the third observation, the length of curves and (locally) the inner distance in $\iota(M)$ defined by the Riemannian metric in $\mathcal{S}$ (equivalently, by the Euclidean metric in $\mathcal{E}$ ) are proportional to that of $M$.

For short, we shall denote

$$
\begin{aligned}
\iota(p) & =\bar{p}, \\
\iota(M) & =\bar{M} .
\end{aligned}
$$

The following notation will be used throughout the paper:

$$
\begin{aligned}
d & =\operatorname{dim} \mathcal{E}-1=\operatorname{dim} \mathcal{S}, \\
c & =\left|\phi_{o}\right|=\sqrt{\phi(o, o)}=\sqrt{d+1}, \\
\mathcal{S}_{u}^{t} & =\{x \in \mathcal{S}:\langle x, u\rangle=t\}, \\
\mathcal{U}_{u}^{t} & =\{x \in \mathcal{S}:\langle x, u\rangle \geq t\},
\end{aligned}
$$

where $u \in \mathcal{E}$ and $t \in \mathbb{R}$. Clearly, for all $u \in \mathcal{S}, p \in M$

$$
|u(p)| \leq c,
$$

where the equality holds only for $u=\bar{p}$. If $t \in[-1,1]$, then we obviously have

$$
\begin{aligned}
& \iota\left(L_{u}^{c t}\right)=\mathcal{S}_{u}^{t} \cap \bar{M}, \\
& \iota\left(U_{u}^{c t}\right)=\mathcal{U}_{u}^{t} \cap \bar{M} .
\end{aligned}
$$

A set which can be realized as a Lipschitz image of a bounded subset of $\mathbb{R}^{k}$ is called $k$-rectifiable (we consider only countable unions of compact sets). If $\iota$ is one-to-one on an $r$-rectifiable set $X \subseteq M$, then, due to (11),

$$
\mathfrak{h}_{\mathcal{S}}^{r}(\iota(X))=s^{r} \mathfrak{h}_{M}^{r}(X) .
$$

In this equality and in the sequel, the Hausdorff measures correspond to the metrics in the related spaces. We shall drop the lower index usually. Let $S^{k}$ be the unit sphere in $\mathbb{R}^{k+1}$. Set

$$
\begin{array}{r}
\varpi_{k}=\mathfrak{h}^{k}\left(S^{k}\right)=\frac{2 \pi^{\frac{k+1}{2}}}{\Gamma\left(\frac{k+1}{2}\right)}, \\
\varkappa_{d}(t)=\mathfrak{h}^{d}\left(\mathcal{U}_{u}^{t}\right)=\varpi_{d-1} \int_{t}^{1}\left(1-\tau^{2}\right)^{\frac{d}{2}-1} d \tau,
\end{array}
$$

where $-1 \leq t \leq 1$. Note that the second term is independent of $u \in \mathcal{S}$ and that $\varkappa_{d}(-1)=\varpi_{d}, \varkappa_{d}(0)=\frac{\varpi_{d}}{2}$. Also, we assume that $\varkappa(t)=\varpi_{d}$ if $t \leq-1$ and $\varkappa(t)=0$ for $t \geq 1$. The definition (5) may be extended onto all level sets:

$$
\mathfrak{l}\left(L_{u}^{t}\right)=\limsup _{\varepsilon \rightarrow+0} \frac{1}{2 \varepsilon} \mathfrak{h}^{m}\left(U_{u}^{t-\varepsilon} \backslash U_{u}^{t+\varepsilon}\right) .
$$


We allow $\mathfrak{r}\left(L_{u}^{t}\right)$ to take the value $+\infty$ and assume that $\mathfrak{r}\left(L_{u}^{t}\right)=0$ if $L_{u}^{t}=\varnothing$. Thus, (16) defines $\mathfrak{l}\left(L_{u}^{t}\right)$ for all $t \in \mathbb{R}$ and $u \in \mathcal{E}$. The quantities $\mathfrak{h}^{m-1}\left(L_{u}^{t}\right)$, $\mathfrak{h}^{m}\left(U_{u}^{t}\right)$, etc., can be extended onto $\mathbb{R} \times \mathcal{E}$ similarly. Since $\left.\mathfrak{h}^{m}\left(U_{u}^{t}\right)\right)$ is piecewise real analytic (see the beginning of the next section), $\mathfrak{l}\left(L_{u}^{t}\right)$ is real analytic outside a finite subset of $\mathbb{R}$. If $t=0$, then $\mathfrak{l}\left(L_{u}^{t}\right)$ is called the Leray measure of the nodal set $N_{u}=L_{u}^{0}$. Since $\mathfrak{h}^{m}\left(U_{u}^{\tau}\right)$ is non-increasing on $\tau$, for almost all $t \in \mathbb{R}$

$$
\mathfrak{l}\left(L_{u}^{t}\right)=-\left.\frac{d}{d \tau} \mathfrak{h}^{m}\left(U_{u}^{\tau}\right)\right|_{\tau=t},
$$

where the derivative exists almost everywhere. Due to the coarea formula (see [9, Theorem 3.2.12]),

$$
\mathfrak{h}^{m}\left(U_{u}^{t}\right)=\int_{t}^{\infty}\left(\int_{L_{u}^{\tau}} \frac{d \mathfrak{h}^{m-1}(p)}{|\nabla u(p)|}\right) d \tau
$$

and almost everywhere on $[-c, c]$ we have

$$
\mathfrak{l}\left(L_{u}^{t}\right)=\int_{L_{u}^{t}} \frac{d \mathfrak{h}^{m-1}(p)}{|\nabla u(p)|} .
$$

The following theorem is a simplified version of Theorem 3.2.48 in [9].

Theorem 1. Let $A, B \subseteq S^{d}$ be compact, $A$ be k-rectifiable, $B$ be $j$-rectifiable, and $\varphi, \psi$ be continuous functions on $A, B$, respectively. Set $r=k+j-d$. Suppose $r \geq 0$. Then

$$
\int_{\mathrm{O}(d+1)} \int_{A \cap g B} \varphi(x) \psi\left(g^{-1} x\right) d \mathfrak{h}^{r}(x) d g=K \int_{A} \varphi(x) d \mathfrak{h}^{k}(x) \int_{B} \psi(x) d \mathfrak{h}^{j}(x),
$$

where $K=\frac{\Gamma\left(\frac{k+1}{2}\right) \Gamma\left(\frac{j+1}{2}\right)}{2 \Gamma\left(\frac{1}{2}\right)^{d} \Gamma\left(\frac{r+1}{2}\right)}=\frac{\varpi_{r}}{\varpi_{k} \varpi_{j}}$.

In particular, for $\varphi=\psi=1$ we have

$$
\int_{\mathrm{O}(d+1)} \mathfrak{h}^{r}(A \cap g B) d g=K \mathfrak{h}^{k}(A) \mathfrak{h}^{j}(B) .
$$

Let us fix an orthogonal decomposition of $\mathcal{E}$ into a sum of $G$-invariant subspaces:

$$
\mathcal{E}=\sum_{j=1}^{l} \oplus \mathcal{E}^{j},
$$

where $\Delta u=-\lambda_{j} u$ for $u \in \mathcal{E}^{j}$. We do not assume that $\lambda_{j} \neq \lambda_{k}$ if $j \neq k$. It follows from (20) that

$$
\phi_{p}=\sum_{j=1}^{l} \phi_{p}^{j},
$$


where $\phi_{p}^{j} \in \mathcal{E}^{j}$ represents the evaluation functional at $p \in M$ on $\mathcal{E}^{j}, j=1, \ldots, l$.

Let each of $\mathcal{E}_{1}, \ldots, \mathcal{E}_{l}$ be as $\mathcal{E}$ above; for $i \in\{1, \ldots, l\}$, we equip the notations of the objects related to $\mathcal{E}_{i}$ with the lower index $i$. Further, we write $\mathbf{t}=$ $\left(t_{1}, \ldots, t_{l}\right) \in \mathbb{R}^{l}$

$$
\begin{aligned}
& \mathbf{E}=\mathcal{E}_{1} \times \cdots \times \mathcal{E}_{l}, \quad \mathbf{S}=\mathcal{S}_{1} \times \cdots \times \mathcal{S}_{l}, \\
& \mathbf{u}=\left(u_{1}, \ldots, u_{l}\right) \in \mathbf{E}, \quad d \mathbf{u}=d u_{1} \ldots d u_{l}, \\
& L_{\mathbf{u}}^{\mathbf{t}}=L_{u_{1}}^{t_{1}} \cap \ldots \cap L_{u_{l}}^{t_{l}}, \quad U_{\mathbf{u}}^{\mathbf{t}}=U_{u_{1}}^{t_{1}} \cap \ldots \cap U_{u_{l}}^{t_{l}},
\end{aligned}
$$

and so on. The mean value (expectation) of a function $f$ on $\mathbf{S}$ or $\mathcal{S}$ is denoted as $\mathrm{M}(f)$ :

$$
\mathbf{M}(f)=\int_{\mathbf{S}} f(\mathbf{u}) d \mathbf{u}, \quad \mathrm{M}(f)=\int_{\mathcal{S}} f(u) d u .
$$

Let $\mathfrak{g}, \mathfrak{h}$ be the Lie algebras of $G, H$ respectively, realized 3 as Lie algebras of vector fields on $M=G / H$ and let $G$ be equipped with a bi-invariant Riemannian metric such that the projection $\eta: G \rightarrow G / H=M$ is a metric submersion. Then $d \eta: \mathfrak{g} \rightarrow T_{o} M$ is isometric on $\mathfrak{h}^{\perp}$, ker $d \eta=\mathfrak{h}$, and $\Delta$ may be defined by (1).

In the following two lemmas, we perform some calculations of [1] in a more general setting. First, we find the coefficient $s=s(\mathcal{E})$ of the local metric homothety $\iota: M \rightarrow \bar{M}$ for $\mathcal{E}$ in (20) (the case of $M=S^{m}$ and one eigenspace was considered in [11]). Set

$$
d_{j}=\operatorname{dim} \mathcal{E}^{j}-1, \quad \alpha_{j}=\frac{d_{j}+1}{d+1}=\frac{\operatorname{dim} \mathcal{E}^{j}}{\operatorname{dim} \mathcal{E}}=\frac{c_{j}^{2}}{c^{2}},
$$

$j=1, \ldots, l$. Thus, $\sum_{j=1}^{l} \alpha_{j}=1$.

Lemma 1. Let $s_{j}=s\left(\mathcal{E}^{j}\right), j=1, \ldots, l$, and $\alpha_{j}$ be as above. Then

$$
s^{2}=\alpha_{1} s_{1}^{2}+\ldots \alpha_{l} s_{l}^{2}=\frac{|\operatorname{Tr} \Delta|}{m \operatorname{dim} \mathcal{E}},
$$

where $\operatorname{Tr} \Delta$ is the trace of $\Delta$ in $\mathcal{E}$.

Proof. Since $\iota$ is equivariant, for all $\xi \in \mathfrak{g}$

$$
d_{o} \iota(\xi(o))=\frac{1}{c} \xi \phi_{o} .
$$

We may choose the basis in (1) such that $\xi_{m+1}, \ldots, \xi_{k} \in \mathfrak{h}$, where $k=\operatorname{dim} \mathfrak{g}$. Then remaining $\xi_{j}$ are orthogonal to $\mathfrak{h}$. Since $\eta: G \rightarrow G / H=M$ is a metric submersion, we have $\left|\xi_{i}(o)\right|=1$ for $i=1, \ldots, m$; clearly, $\xi_{i}(o)=0$ for $i=$ $m+1, \ldots, k$. Using consequently (11), (24), (10), (11), and (21), we get

$$
\begin{array}{r}
m s^{2}=s^{2} \sum_{i=1}^{k}\left|\xi_{i}(o)\right|^{2}=\sum_{i=1}^{k}\left|d_{o} \iota\left(\xi_{i}(o)\right)\right|^{2}=\frac{1}{c^{2}} \sum_{i=1}^{k}\left|\xi_{i} \phi_{o}\right|^{2}=-\frac{1}{c^{2}} \sum_{i=1}^{k}\left\langle\xi_{i}^{2} \phi_{o}, \phi_{o}\right\rangle \\
=-\frac{1}{c^{2}}\left\langle\Delta \phi_{o}, \phi_{o}\right\rangle=\frac{1}{c^{2}}\left\langle\sum_{j=1}^{l} \lambda_{j} \phi_{o}^{j}, \phi_{o}\right\rangle=\frac{1}{c^{2}} \sum_{j=1}^{l} \lambda_{j}\left|\phi_{o}^{j}\right|^{2}=\sum_{j=1}^{l} \alpha_{j} \lambda_{j} .
\end{array}
$$

\footnotetext{
${ }^{3}$ we assume that the action of $G$ is virtually effective; see the footnote on page 2
} 
If $l=1$, then $s=\sqrt{\frac{\lambda_{1}}{m}}$; hence, $s_{j}=\sqrt{\frac{\lambda_{j}}{m}}$. This proves the first equality in (23); the second is true since $\left|\phi_{o}^{j}\right|^{2}=\operatorname{dim} \mathcal{E}^{j}=c_{j}^{2}, c^{2}=\operatorname{dim} \mathcal{E}$.

The second lemma is similar to Lemma 6 of the paper [11, where (25) was proved for $t=0$.

Lemma 2. Let $|t| \leq 1$ and $X \subseteq M$ be $(r+1)$-rectifiable, where $r \leq m-1$. Then

$$
\begin{array}{r}
\int_{\mathcal{S}} \mathfrak{h}^{r}\left(L_{u}^{c t} \cap X\right) d u=\frac{\varpi_{r}}{\varpi_{r+1}} s \mathfrak{h}^{r+1}(X)\left(1-t^{2}\right)^{\frac{d-1}{2}}, \\
\int_{\mathcal{S}} \mathfrak{h}^{r+1}\left(U_{u}^{c t} \cap X\right) d u=\mathfrak{h}^{r+1}(X) \frac{\varkappa_{d}(t)}{\varpi_{d}} .
\end{array}
$$

Proof. Since both sides of (25) are additive on $X$ and $\iota$ is a finite covering, it is sufficient to prove (25) assuming that $\iota$ is injective on $X$. For each $t \in[-1,1]$, the group $O(\mathcal{E})$ acts transitively on the family of spheres $\left\{\mathcal{S}_{u}^{t}\right\}_{u \in \mathcal{S}}$. Due to (13), we may apply Theorem 1 to $\mathcal{S}$ setting $A=\mathcal{S}_{o}^{t}, k=d-1, B=\iota(X), j=r+1$ in (19). Since the Euclidean radius of $\mathcal{S}_{\bar{o}}^{t}$ is equal to $\sqrt{1-t^{2}}$, we have

$$
\mathfrak{h}^{d-1}\left(\mathcal{S}_{\bar{o}}^{t}\right)=\varpi_{d-1}\left(1-t^{2}\right)^{\frac{d-1}{2}} .
$$

Using (13), (15), replacing integration over $\mathcal{S}$ with averaging over $\mathrm{O}(\mathcal{E})$, and applying (19), we get (25):

$$
\begin{array}{r}
\int_{\mathcal{S}} \mathfrak{h}^{r}\left(L_{u}^{c t} \cap X\right) d u=\frac{1}{s^{r}} \int_{\mathcal{S}} \mathfrak{h}^{r}\left(\iota\left(L_{u}^{c t} \cap X\right)\right) d u=\frac{1}{s^{r}} \int_{\mathcal{S}} \mathfrak{h}^{r}\left(\mathcal{S}_{u}^{t} \cap \iota(X)\right) d u \\
=\frac{1}{s^{r}} \int_{\mathrm{O}(\mathcal{E})} \mathfrak{h}^{r}\left(g \mathcal{S}_{\bar{o}}^{t} \cap \iota(X)\right) d g=\frac{1}{s^{r}} K \mathfrak{h}^{d-1}\left(\mathcal{S}_{\bar{o}}^{t}\right) \mathfrak{h}^{r+1}(\iota(X)) \\
=\frac{\varpi_{r}}{s^{r} \varpi_{r+1}}\left(1-t^{2}\right)^{\frac{d-1}{2}} \mathfrak{h}^{r+1}(\iota(X))=s\left(1-t^{2}\right)^{\frac{d-1}{2}} \frac{\varpi_{r}}{\varpi_{r+1}} \mathfrak{h}^{r+1}(X) .
\end{array}
$$

To prove (26), set $A=\mathcal{U}_{\bar{o}}^{t}, k=d, B=\iota(X), j=r+1$ in (19). Then

$$
\begin{gathered}
\int_{\mathcal{S}} \mathfrak{h}^{r+1}\left(U_{u}^{c t} \cap X\right) d u=\frac{1}{s^{r+1}} \int_{\mathrm{O}(\mathcal{E})} \mathfrak{h}^{r+1}\left(g \mathcal{U}_{\bar{o}}^{t} \cap \iota(X)\right) d g \\
=\frac{1}{s^{r+1}} \frac{\varpi_{r+1}}{\varpi_{d} \varpi_{r+1}} \varkappa_{d}(t) \mathfrak{h}^{r+1}(\iota(X))=\frac{\varkappa_{d}(t)}{\varpi_{d}} \mathfrak{h}^{r+1}(X),
\end{gathered}
$$

where we omit some steps since they are similar to those above.

\section{Computation of expectations}

We formulate below two Łojasiewicz's inequalities following [2] but in a weaker form (cf. Theorem 6.4, Remark 6.5, and Proposition 6.8 of [2]). Let $\mathrm{CV}_{N}(f)$ denote the set of critical values of a smooth function $f$ on a manifold $N$ (we 
drop the index if no confusion can occur). Suppose $f$ real analytic in a domain $\mathcal{D} \subseteq \mathbb{R}^{n}, N_{f}=f^{-1}(0)$. Let $Q$ be a compact subset of $\mathcal{D}$. Then there exist $\nu, \eta>0$ such that

$$
|f(q)| \geq \eta \operatorname{dist}\left(q, N_{f}\right)^{\nu}
$$

for all $q \in Q$, where dist denotes the Euclidean distance. Furthermore, for any $x \in N_{f}$ there are its neighborhood $U$ and $\eta>0, \theta \in(0,1)$ such that for all $q \in U$

$$
|\nabla f(q)| \geq \eta|f(q)|^{\theta},
$$

where $\nabla$ stands for the Euclidean gradient.

Lemma 3. For all $u \in \mathcal{S}$

(i) the set $\mathrm{CV}(u)$ is finite,

(ii) $\mathfrak{h}^{m-1}\left(L_{u}^{t}\right)$ and $\mathfrak{l}\left(L_{u}^{t}\right)$ are smooth on $u(M) \backslash \mathrm{CV}(u)$ as functions of $t$,

(iii) for any $t_{0} \in \mathrm{CV}(u)$ there are $\theta \in(0,1)$ and $\eta>0$ such that

$$
\mathfrak{l}\left(L_{u}^{t}\right) \leq \eta \mathfrak{h}^{m-1}\left(L_{u}^{t}\right)\left|t-t_{0}\right|^{-\theta},
$$

where $t \in u(M) \backslash \mathrm{CV}(u)$.

Proof. (i). The set of critical points of $u$ is defined by the equation $|\nabla u(x)|^{2}=0$. Hence, it has a finite number of components being an analytic set in a compact manifold (moreover, $\iota$ maps this set onto a real algebraic set in $\mathcal{E}$ since $\bar{M}$ can be distinguished in $\mathcal{E}$ by $G$-invariant polynomials). On the other hand, the set $\mathrm{CV}(u)$ has zero Lebesgue measure in $\mathbb{R}$ by Sard's theorem since $u$ is sufficiently smooth. Hence $u$ is constant on each component.

(ii). By (i), the set $u(M) \backslash \mathrm{CV}(u)$ is the union of a finite family of disjoint open intervals. Let $I$ be a compact subinterval in $u(M) \backslash \mathrm{CV}(u)$ and let $t \in I$. By a basic theorem of Morse Theory, $u^{-1}(I)$ is diffeomorphic to $I \times N$, where $N=u^{-1}(t)$ is a smooth submanifold of $M$ (see [26, Theorem 3.1] or [29, 9.3.3]). Together with the coarea formula and (18), this implies (ii).

(iii). Set $f(p)=u(p)-t_{0}$. Every point in the critical level set $L_{u}^{t_{0}}$ has a neighborhood in $M$ where (28) holds. Standard compactness arguments and (i) show that (28) is true in some neighborhood of $L_{u}^{t_{0}}$ in $M$ (we may assume that $|f(p)|<1$ in every neighborhood, then we may increase $\theta$ keeping the inequality (28) and the inclusion $\theta \in(0,1))$. Applying (18) with $t=u(p)$, we get (29) in some neighborhood of $t_{0}$; it admits an extension onto $u(M)$ with, may be, a smaller $\eta$.

In the following theorem, we use the notation of (22). For a function $f$ on $[-c, c]$ set

$$
I_{f}(u)=\int_{M} f(u(p)) d p
$$


Theorem 2. Let all factors in $\mathbf{E}$ satisfy $(\mathrm{E}), X \subseteq M$ be r-rectifiable for some integer $r, 1 \leq r \leq m, l \in \mathbb{N}, \mathbf{t}=\left(t_{1}, \ldots, t_{l}\right)$, and $t_{i} \in\left[-c_{i}, c_{i}\right]$ for all $i=1, \ldots, l$.

(1) If $l \leq r$, then

$$
\mathrm{M}\left(\mathfrak{h}^{r-l}\left(L_{\mathbf{u}}^{\mathbf{t}} \cap X\right)\right)=\frac{\varpi_{r-l}}{\varpi_{r}} \mathfrak{h}^{r}(X) \prod_{i=1}^{l} s_{i}\left(1-\frac{t_{i}^{2}}{c_{i}^{2}}\right)^{\frac{d_{i}-1}{2}},
$$

where $s_{i}$ is subject to (23) with $\mathcal{E}=\mathcal{E}_{i}, i=1, \ldots, l$.

(2) For any $l \in \mathbb{N}$

$$
\mathrm{M}\left(\mathfrak{h}^{r}\left(U_{\mathbf{u}}^{\mathbf{t}} \cap X\right)\right)=\mathfrak{h}^{r}(X) \prod_{i=1}^{l} \frac{\varkappa_{d_{i}}\left(\frac{t_{i}}{c_{i}}\right)}{\varpi_{d_{i}}} .
$$

(3) For almost all $t \in[-c, c]$

$$
\mathrm{M}\left(\mathfrak{l}\left(L_{u}^{t}\right)\right)=\frac{\varpi \varpi_{d-1}}{c \varpi_{d}}\left(1-\frac{t^{2}}{c^{2}}\right)^{\frac{d}{2}-1} .
$$

Moreover, if $f$ is a piecewise continuous function on $[-c, c]$, then

$$
\mathrm{M}\left(I_{f}\right)=\frac{\varpi \varpi_{d-1}}{c \varpi_{d}} \int_{-c}^{c} f(t)\left(1-\frac{t^{2}}{c^{2}}\right)^{\frac{d}{2}-1} d t .
$$

Proof. (1). Applying (25) repeatedly, we get (31):

$$
\begin{gathered}
\int_{\mathbf{S}} \mathfrak{h}^{r-l}\left(L_{\mathbf{u}}^{\mathbf{t}} \cap X\right) d \mathbf{u} \\
=\int_{\mathcal{S}_{2} \times \cdots \times \mathcal{S}_{l}}\left(\int_{\mathcal{S}_{1}} \mathfrak{h}^{r-l}\left(L_{u_{1}}^{t_{1}} \cap\left(L_{u_{2}}^{t_{2}} \cap \ldots \cap L_{u_{l}}^{t_{l}} \cap X\right)\right) d u_{1}\right) d u_{2} \ldots d u_{l} \\
=\frac{\varpi_{r-l}}{\varpi_{r-l+1}} s_{1}\left(1-\frac{t_{1}^{2}}{c_{1}^{2}}\right)^{\frac{d_{1}-1}{2}} \int_{\mathcal{S}_{2} \times \cdots \times \mathcal{S}_{l}} \mathfrak{h}^{r-l+1}\left(L_{u_{2}}^{t_{2}} \cap \ldots \cap L_{u_{l}}^{t_{l}} \cap X\right) d u_{2} \ldots d u_{l} \\
\quad=\cdots=\frac{\varpi_{r-l}}{\varpi_{r}} \mathfrak{h}^{r}(X) \prod_{i=1}^{l} s_{i}\left(1-\frac{t_{i}^{2}}{c_{i}^{2}}\right)^{\frac{d_{i}-1}{2}} .
\end{gathered}
$$

(2). Similarly, by (26),

$$
\begin{array}{r}
\int_{\mathbf{S}} \mathfrak{h}^{m}\left(U_{\mathbf{u}}^{\mathbf{t}} \cap X\right) d \mathbf{u}=\frac{\varkappa_{d_{1}}\left(\frac{t_{1}}{c_{1}}\right)}{\varpi_{d_{1}}} \int_{\mathcal{S}_{2} \times \cdots \times \mathcal{S}_{l}} \mathfrak{h}^{m}\left(U_{u_{2}}^{t_{2}} \cap \ldots \cap U_{u_{l}}^{t_{l}}\right) d u_{2} \ldots d u_{l}=\ldots \\
=\varpi \prod_{i=1}^{l} \frac{\varkappa_{d_{i}}\left(\frac{t_{i}}{c_{i}}\right)}{\varpi_{d_{i}}} .
\end{array}
$$


This proves (32).

(3). The equality (7) is a particular case of (32) for $r=m$ and $X=M$. By (7), the right-hand side of (33) is equal to $-\frac{d}{d t} \mathrm{M}\left(\mathfrak{h}^{m}\left(U_{u}^{t}\right)\right)$. According to (16), we have to check the equality

$$
\int_{\mathcal{S}} \frac{d}{d t} \mathfrak{h}^{m}\left(U_{u}^{t}\right) d u=\frac{d}{d t} \mathbf{M}\left(\mathfrak{h}^{m}\left(U_{u}^{t}\right)\right) .
$$

for almost all $t$. We claim that for any fixed $u \in \mathcal{S}$ the function $\mathfrak{h}^{m}\left(U_{u}^{t}\right)$ is absolutely continuous on $t$ in the interval $u(M)$. Indeed, on the set $u(M) \backslash \mathrm{CV}(u)$ this is true according to Lemma 3. (ii); since this function is non-increasing and $\mathrm{CV}(u)$ is finite, it is sufficient to prove that it is continuous. We have

$$
\lim _{\varepsilon \rightarrow 0} \mathfrak{h}^{m}\left(U_{u}^{t-\varepsilon} \backslash U_{u}^{t+\varepsilon}\right)=\mathfrak{h}^{m}\left(L_{u}^{t}\right)=0,
$$

where the first equality is evident and the second holds because $u$ is real analytic on $M: \mathfrak{h}^{m}\left(L_{u}^{t}\right)>0$ implies $u=t$, contradictory to the assumption in (E) that $\mathcal{E}$ is orthogonal to constant functions since $u \neq 0$ due to the inclusion $u \in \mathcal{S}$. (The implication is obvious if $m=1$; for $m>1$ one can use the immersion $\iota$ to prove that a set of positive $\mathfrak{h}^{m}$-measure in $M$ intersects sufficiently many real analytic curves (preimages of the big circles) in sets of positive $\mathfrak{h}^{1}$-measure.)

Thus, $\mathfrak{h}^{m}\left(U_{u}^{t}\right)$ is absolutely continuous on $[-c, c]$ and we may apply the Newton-Leibnitz formula on $t$ to $\mathfrak{l}\left(L_{u}^{t}\right)$ on any subinterval $[a, b]$ of $[-c, c]$. Since it is nonnegative and has variation $\varpi$ on $[-c, c], \mathfrak{l}\left(L_{u}^{t}\right)$ is summable on $\mathcal{S} \times[-c, c]$. In particular, $\mathrm{M}\left(\mathfrak{l}\left(L_{u}^{t}\right)\right)$ is well defined for almost all $t$. By Fubini's theorem,

$$
\int_{a}^{b} \mathrm{M}\left(\mathfrak{l}\left(L_{u}^{t}\right)\right) d t=\mathrm{M}\left(\int_{a}^{b} \mathfrak{l}\left(L_{u}^{t}\right) d t\right)=\mathrm{M}\left(\mathfrak{h}^{m}\left(U_{u}^{a}\right)\right)-\mathrm{M}\left(\mathfrak{h}^{m}\left(U_{u}^{b}\right)\right) .
$$

Thus, the integrals of the left-hand and the right-hand parts of (35) over any subinterval in $[-c, c]$ coincide; hence, (35) holds almost everywhere on $t$. This proves (33).

Since $\mathfrak{h}^{m}\left(U_{u}^{t}\right)$ is absolutely continuous, we may apply Fubini's theorem to the equality

$$
\mathrm{M}\left(I_{f}\right)=\int_{\mathcal{S}}\left(\int_{-c}^{c} f(t) \mathfrak{r}\left(L_{u}^{t}\right) d t\right) d u .
$$

Thus, (34) follows from (33).

Remark 2. Note that the right-hand side of (7), as well as (26) and (32), depends only on $d$ and $\varpi$. Thus, for isotropy irreducible homogeneous spaces, the expectations of $\mathfrak{h}^{m}\left(U_{u}^{t}\right)$ are independent of their topology and of the spectrum of $\Delta$ in $\mathcal{E}$. According to (16), the same is true for the Leray measure. The corollary below shows that the asymptotic behavior of the expectations as $\operatorname{dim} \mathcal{E} \rightarrow \infty$ is independent of the choice of subspaces $\mathcal{E} \subset L^{2}(M)$, as well as of $M$, except for the left-hand side of (37). 
Corollary 1. Let $\mathcal{E}_{n}$ be a sequence of subspaces of $L^{2}(M)$ which satisfy $(\mathrm{E})$. Suppose $d_{n} \rightarrow \infty$ as $n \rightarrow \infty$. Then for any $t \in \mathbb{R}$ and r-rectifiable $X \subseteq M$, where $r \leq m$, we have

$$
\begin{array}{r}
\lim _{n \rightarrow \infty} \frac{1}{s_{n}} \mathrm{M}\left(\mathfrak{h}^{r-1}\left(L_{u}^{t} \cap X\right)\right)=\frac{\varpi_{r-1}}{\varpi_{r}} \mathfrak{h}^{r}(X) e^{-\frac{t^{2}}{2}}, \\
\lim _{n \rightarrow \infty} \mathrm{M}\left(\mathfrak{h}^{r}\left(U_{u}^{t} \cap X\right)\right)=\frac{\mathfrak{h}^{r}(X)}{\sqrt{\pi}} \operatorname{erfc}\left(\frac{t}{\sqrt{2}}\right), \\
\lim _{n \rightarrow \infty} \mathrm{M}\left(\mathfrak{l}\left(L_{u}^{t}\right)\right)=\frac{\varpi}{\sqrt{2 \pi}} e^{-\frac{t^{2}}{2}},
\end{array}
$$

where $\operatorname{erfc}(t)=\int_{t}^{\infty} e^{-\tau^{2}} d \tau$. If $f$ is a piecewise continuous function on $\mathbb{R}$ such that $\int_{-\infty}^{\infty}|f(t)| e^{-\frac{t^{2}}{2}} d t<\infty$, then

$$
\lim _{n \rightarrow \infty} \mathrm{M}\left(I_{f}\right)=\frac{\varpi}{\sqrt{2 \pi}} \int_{-\infty}^{\infty} f(t) e^{-\frac{t^{2}}{2}} d t
$$

Proof. Since $c_{n}^{2}=d_{n}+1$, we have $\lim _{n \rightarrow \infty}\left(1-\frac{t^{2}}{c_{n}^{2}}\right)^{\frac{d_{n}-1}{2}}=e^{-\frac{t^{2}}{2}}$. Together with the theorem for $l=1$, this implies (37) and (38). Taking in account the equality

$$
\lim _{n \rightarrow \infty} \frac{\varpi_{d_{n}-1}}{c_{n} \varpi_{d_{n}}}=\lim _{n \rightarrow \infty} \frac{\Gamma\left(\frac{d_{n}+1}{2}\right)}{\sqrt{\pi} c_{n} \Gamma\left(\frac{d_{n}}{2}\right)}=\frac{1}{\sqrt{2 \pi}},
$$

we get (39) and (40).

The results above make it possible to find expectations for radial distributions on $\mathcal{E}$. Let $\alpha$ be a nonnegative measurable function on $[0, \infty)$ such that $\alpha \neq 0$ and

$$
a_{k}=\int_{0}^{\infty} r^{k} \alpha(r) d r<\infty
$$

for all $k \in \mathbb{N}$. It defines a probability measure $\alpha_{d}(x) d x$ on $\mathcal{E}$, where $d x$ stands for the Lebesgue measure on $\mathcal{E}$, with the density

$$
\alpha_{d}(x)=\frac{1}{a_{d} \varpi_{d}} \alpha(|x|) .
$$

We denote the mean value of a function $f$ on $\mathcal{E}$ with respect to a probability measure $\eta(x) d x$ as

$$
\mathrm{M}^{\eta}(f)=\int_{\mathcal{E}} f(x) \eta(x) d x
$$

Since $U_{u}^{t} \cup U_{-u}^{-t}=M, U_{u}^{t} \cap U_{-u}^{-t}=L_{u}^{t}$, and $u$ is real analytic, for $u \neq 0$ we have

$$
\mathfrak{h}^{m}\left(U_{u}^{t}\right)+\mathfrak{h}^{m}\left(U_{-u}^{-t}\right)=\varpi .
$$

Hence we may assume $t \geq 0$. 
Proposition 1. Let $\alpha, a_{d}$, and $\alpha_{d}$ be as above and $t \geq 0$. Then

$$
\begin{array}{r}
\mathrm{M}^{\alpha_{d}}\left(\mathfrak{h}^{m-1}\left(L_{u}^{c t}\right)\right)=\frac{\varpi \varpi_{m-1} s}{a_{d} \varpi_{m}} \int_{t}^{\infty}\left(r^{2}-t^{2}\right)^{\frac{d-1}{2}} r \alpha(r) d r, \\
\mathrm{M}^{\alpha_{d}}\left(\mathfrak{h}^{m}\left(U_{u}^{c t}\right)\right)=\frac{\varpi \varpi_{d-1}}{2 a_{d} \varpi_{d}} \int_{t}^{\infty}\left(\int_{0}^{\infty} \tau^{\frac{d}{2}-1} \alpha\left(\sqrt{\tau+\xi^{2}}\right) d \tau\right) d \xi, \\
\mathrm{M}^{\alpha_{d}}\left(\mathfrak{l}\left(L_{u}^{c t}\right)\right)=\frac{\varpi \varpi_{d-1}}{2 c a_{d} \varpi_{d}} \int_{0}^{\infty} \tau^{\frac{d}{2}-1} \alpha\left(\sqrt{\tau+t^{2}}\right) d \tau .
\end{array}
$$

Proof. Let $\mathcal{S}_{r}$ denote the sphere of radius $r$ centered at zero (thus $\mathcal{S}=\mathcal{S}_{1}$ ) and $d u$ be the invariant probability measure on $\mathcal{S}_{r}$. We have

$$
\int_{\mathcal{E}} \mathfrak{h}^{m-1}\left(L_{u}^{c t}\right) \alpha(|x|) d x=\varpi_{d} \int_{0}^{\infty}\left(\int_{\mathcal{S}_{r}} \mathfrak{h}^{m-1}\left(L_{u}^{c t}\right) d u\right) r^{d} \alpha(r) d r .
$$

Clearly,

$$
\int_{\mathcal{S}_{r}} f(u) d u=\int_{\mathcal{S}} f(r u) d u
$$

for any continuous function $f$ on $\mathcal{E}$. Furthermore, $L_{u}^{t}=L_{r u}^{r t}$ for all $r>0$. If $|u|<t$, then $L_{u}^{c t}=\varnothing$ by (12). Thus, the integral in the right-hand side of (45) is equal to

$$
\begin{aligned}
\int_{t}^{\infty}\left(\int_{\mathcal{S}_{r}} \mathfrak{h}^{m-1}\left(L_{u}^{c t}\right) d u\right) r^{d} \alpha(r) d r=\int_{t}^{\infty} & \left(\int_{\mathcal{S}} \mathfrak{h}^{m-1}\left(L_{u}^{\frac{c t}{r}}\right) d u\right) r^{d} \alpha(r) d r \\
= & \int_{t}^{\infty} \mathrm{M}\left(\mathfrak{h}^{m-1}\left(L_{u}^{\frac{c t}{r}}\right)\right) r^{d} \alpha(r) d r .
\end{aligned}
$$

Using (6), we obtain (42) by a straightforward calculation. The expectations of $\mathfrak{h}^{m}\left(U_{u}^{c t}\right)$ and $\mathfrak{l}\left(L_{u}^{c t}\right)$ can be calculated similarly: since $U_{u}^{t}=U_{r u}^{r t}$ for any $r>0$ and $U_{u}^{c t}=\varnothing$ if $|u|<t$, (46) and (77) imply

$$
\begin{aligned}
\mathrm{M}^{\alpha_{d}}\left(\mathfrak{h}^{m}\left(U_{u}^{c t}\right)\right) & =\frac{1}{a_{d}} \int_{t}^{\infty}\left(\int_{\mathcal{S}} \mathfrak{h}^{m}\left(U_{u}^{\frac{c t}{r}}\right) d u\right) r^{d} \alpha(r) d r \\
& =\frac{\varpi \varpi_{d-1}}{a_{d} \varpi_{d}} \int_{t}^{\infty}\left(\int_{\frac{t}{r}}^{1}\left(1-\eta^{2}\right)^{\frac{d}{2}-1} d \eta\right) r^{d} \alpha(r) d r .
\end{aligned}
$$

Let us change the order of integration and substitute $\xi=\frac{\eta}{r}$ :

$\int_{t}^{\infty}\left(\int_{t}^{r}\left(r^{2}-\xi^{2}\right)^{\frac{d}{2}-1} d \xi\right) r \alpha(r) d r=\int_{t}^{\infty}\left(\int_{\xi}^{\infty}\left(r^{2}-\xi^{2}\right)^{\frac{d}{2}-1} r \alpha(r) d r\right) d \xi$.

Using the change of variable $\tau=r^{2}-\xi^{2}$, we get (43) and, by differentiation of (43) on $t$, (44). 
Corollary 2. If $\alpha_{d}$ is the Gaussian density on $\mathcal{E}$ defined by (41) for $\alpha(t)=$ $G^{\sigma}(t)=e^{-\frac{t^{2}}{\sigma^{2}}}$, then

$$
\begin{aligned}
\mathrm{M}^{\alpha_{d}}\left(\mathfrak{h}^{m-1}\left(L_{u}^{c t}\right)\right) & =\varpi \frac{\varpi_{m-1}}{\varpi_{m}} s e^{-\frac{t^{2}}{\sigma^{2}}}, \\
\mathrm{M}^{\alpha_{d}}\left(\mathfrak{h}^{m}\left(U_{u}^{c t}\right)\right) & =\frac{\varpi}{\sqrt{\pi}} \operatorname{erfc}\left(\frac{t}{\sigma}\right), \\
\mathrm{M}^{\alpha_{d}}\left(\mathfrak{l}\left(L_{u}^{c t}\right)\right) & =\frac{\varpi}{c \sigma \sqrt{\pi}} e^{-\frac{t^{2}}{\sigma^{2}}},
\end{aligned}
$$

where $\operatorname{erfc}(t)=\int_{t}^{\infty} e^{-\tau^{2}} d \tau$.

Proof. We have $a_{d}=\frac{\sigma^{d+1}}{2} \Gamma\left(\frac{d+1}{2}\right)$. By (42),

$$
\begin{aligned}
\mathbf{M}^{\alpha_{d}}\left(\mathfrak{h}^{m-1}\left(L_{u}^{c t}\right)\right) & =\frac{\varpi \varpi_{m-1} s}{a_{d} \varpi_{m}} \int_{t}^{\infty}\left(r^{2}-t^{2}\right)^{\frac{d-1}{2}} r e^{-\frac{r^{2}}{\sigma^{2}}} d r \\
& =\frac{\varpi \varpi_{m-1} s}{\Gamma\left(\frac{d+1}{2}\right) \varpi_{m}} e^{-\frac{t^{2}}{\sigma^{2}}} \int_{0}^{\infty} \tau^{\frac{d-1}{2}} e^{-\tau} d \tau=\varpi \frac{\varpi_{m-1}}{\varpi_{m}} s e^{-\frac{t^{2}}{\sigma^{2}}},
\end{aligned}
$$

where $\tau=\frac{r^{2}-t^{2}}{\sigma^{2}}$. Further, $\frac{\varpi_{d-1}}{a_{d} \varpi_{d}}=\frac{2}{\sqrt{\pi} \sigma^{d+1} \Gamma\left(\frac{d}{2}\right)}$; therefore,

$$
\begin{aligned}
\mathrm{M}^{\alpha_{d}} & \left(\mathfrak{h}^{m-1}\left(U_{u}^{c t}\right)\right)=\frac{\varpi}{\sigma^{d+1} \sqrt{\pi} \Gamma\left(\frac{d}{2}\right)} \int_{t}^{\infty}\left(\int_{0}^{\infty} \tau^{\frac{d}{2}-1} e^{-\frac{\tau}{\sigma^{2}}} d \tau\right) e^{-\frac{\xi^{2}}{\sigma^{2}}} d \xi \\
= & \frac{\varpi}{\sigma \sqrt{\pi} \Gamma\left(\frac{d}{2}\right)} \int_{t}^{\infty}\left(\int_{0}^{\infty} \eta^{\frac{d}{2}-1} e^{-\eta} d \eta\right) e^{-\frac{\xi^{2}}{\sigma^{2}}} d \xi=\frac{\varpi}{\sqrt{\pi}} \operatorname{erfc}\left(\frac{t}{\sigma}\right) .
\end{aligned}
$$

Differentiating on $t$, we get the last equality of the corollary.

Remark 3. According to (39)), $\lim _{d \rightarrow \infty} \mathrm{M}\left(\mathfrak{l}\left(N_{u}\right)\right)=\frac{w}{\sqrt{2 \pi}}$. In the papers 28. and [39], the expectations of $\mathfrak{r}\left(N_{u}\right)$ were computed for tori $\mathbb{R}^{n} / \mathbb{Z}^{n}$ and spheres $S^{m}$ with the Gaussian distribution in $\mathcal{E}$ normalized by the condition that for any fixed $p \in M$ the average of $|u(p)|^{2}$ is equal to 1 . In both cases, the expectation is independent of $\operatorname{dim} \mathcal{E}$ and equals to $\frac{\varpi}{\sqrt{2 \pi}}$, where $\varpi=1$ for $\mathbb{R}^{n} / \mathbb{Z}^{n}$ and $\varpi=\varpi_{m}$ for $S^{m}$. By a direct computation one can check that this is equivalent to the relation $\sigma c=\sqrt{2}$ in the notation of Corollary 2 It follows from Corollary 2 that the same is true for all isotropy irreducible homogeneous spaces: the expectation of $\mathfrak{l}\left(N_{u}\right)$ for the Gaussian distribution with this normalization is equal to $\frac{\varpi}{\sqrt{2 \pi}}$ independently of $\mathcal{E}$. For the uniform distribution on spheres the expectation depends on $\operatorname{dim} \mathcal{E}$ but mildly since $\frac{\varpi_{d-1}}{c_{d}} \rightarrow \frac{1}{\sqrt{2 \pi}}$ as $d \rightarrow \infty$.

\section{Upper bounds for the expectations of $L^{p}$ norms}

We use the symbol $a$ instead of the standard $p$ in $\|u\|_{a}$ (the norm in the spaces $\left.L^{a}(M), 1 \leq a \leq \infty\right)$. There are two reasons for it: first, $p$ denotes a point of $M$ in the text above, and second, we do not exclude the cases $a \in(0,1)$ and even $a \in(-1,0)$ in the calculation below. 
Theorem 3. Let $a>-1$. The function $|u|^{a}$ is integrable on $M$ for almost all $u \in \mathcal{S}$. Moreover, $\int_{M}|u(p)|^{a} d p \in L^{1}(\mathcal{S})$ and

$$
\mathrm{M}\left(\int_{M}|u(p)|^{a} d p\right)=\frac{\Gamma\left(\frac{a+1}{2}\right) \Gamma\left(\frac{d+1}{2}\right)(d+1)^{\frac{a}{2}}}{\sqrt{\pi} \Gamma\left(\frac{a+d+1}{2}\right)} .
$$

If $a>2$ or $a \in(-1,0)$, then for all $d \in \mathbb{N}$

$$
\mathrm{M}\left(\int_{M}|u(p)|^{a} d p\right)<2^{\frac{a}{2}} \frac{\Gamma\left(\frac{a+1}{2}\right)}{\sqrt{\pi}},
$$

the reverse inequality holds for $a \in(0,2)$, and the equality is true if $a=0$ or $a=2$.

Proof. If $a>0$, then we may apply (34) to $f(t)=|t|^{a}$ :

$$
\begin{gathered}
\mathrm{M}\left(I_{f}\right)=c^{a} \frac{\varpi_{d-1}}{\varpi_{d}} \int_{-1}^{1}|t|^{a}\left(1-t^{2}\right)^{\frac{d}{2}-1} d t=c^{a} \frac{\varpi_{d-1}}{\varpi_{d}} \int_{0}^{1} \tau^{\frac{a-1}{2}}(1-\tau)^{\frac{d}{2}-1} d \tau \\
=c^{a} \frac{\varpi_{d-1}}{\varpi_{d}} B\left(\frac{a+1}{2}, \frac{d}{2}\right)=c^{a} \frac{\varpi_{d-1}}{\varpi_{d}} \frac{\Gamma\left(\frac{d}{2}\right) \Gamma\left(\frac{a+1}{2}\right)}{\Gamma\left(\frac{a+d+1}{2}\right)}=\frac{\Gamma\left(\frac{a+1}{2}\right) \Gamma\left(\frac{d+1}{2}\right)}{\sqrt{\pi} \Gamma\left(\frac{a+d+1}{2}\right)} c^{a} .
\end{gathered}
$$

All equalities, with the possible exception for the first, hold true for $a \in(-1,0)$. Hence, we get the same result for such $a$ approximating $|t|^{a}$ by the functions $f_{n}(t)=\min \left\{|t|^{a}, n\right\}$. Indeed, the sequence $I_{f_{n}}(u)$ increases for any $u \in \mathcal{S}$ and $f_{n}(t)$ converges to $|t|^{a}$ if $t \neq 0$. Hence

$$
\frac{c \varpi_{d}}{\varpi \varpi_{d-1}} \mathrm{M}\left(I_{f_{n}}\right)=\int_{-c}^{c} f_{n}(t)\left(1-\frac{t^{2}}{c^{2}}\right)^{\frac{d}{2}-1} d t \rightarrow \int_{-c}^{c}|t|^{a}\left(1-\frac{t^{2}}{c^{2}}\right)^{\frac{d}{2}-1} d t
$$

as $n \rightarrow \infty$. It follows from Levy's and Lebesgue's theorems that $I_{f_{n}}(u) \rightarrow$ $\int_{M}|u(p)|^{a} d p$ and $|u|^{a} \in L^{1}(M)$ for almost all $u \in \mathcal{S}$. Thus, $\int_{M}|u(p)|^{a} d p \in$ $L^{1}(\mathcal{S})$. This verifies the calculation. The inequalities follow from Lemma 4 below, the cases $a=0$ and $a=2$ are obvious.

For the sake of completeness, we give a proof of some properties of Euler's function $\Gamma$.

Lemma 4. Set $\varphi_{b}(t)=\frac{t^{b} \Gamma(t)}{\Gamma(t+b)}, f(t)=\ln \left(\left(\frac{e}{t}\right)^{t-\frac{1}{2}} \Gamma(t)\right)$.

(a) The function $\varphi_{b}$ decreases on $(0, \infty)$ if $0<b<1$ and increases if $b>$ 1. Moreover, if $b<0$, then $\varphi_{b}$ increases on $(-b, \infty)$. For any $b \in \mathbb{R}$ $\lim _{t \rightarrow \infty} \varphi_{b}(t)=1$.

(b) The function $f$ is convex on $(0, \infty)$ and $\lim _{t \rightarrow \infty} f(t)=\ln \sqrt{\frac{2 \pi}{e}}$.

(c) For all $t \in\left(\frac{1}{2}, \infty\right)$

$$
1>\left(\frac{e}{t}\right)^{t-\frac{1}{2}} \frac{\Gamma(t)}{\sqrt{\pi}}>\sqrt{\frac{2}{e}}
$$


Proof. The limit in (a) follows from the Stirling formula. To prove the first and the second assertions in (a), let us consider $\Psi(x)=\frac{d}{d x} \ln \Gamma(x)$. We have

$$
\Psi^{\prime \prime}(x)=-2 \sum_{k=0}^{\infty} \frac{1}{(x+k)^{3}}<0
$$

for all $x>0$. Hence the function

$$
\eta_{t}(b)=\frac{d}{d t} \ln \varphi_{b}(t)=\frac{b}{t}+\Psi(t)-\Psi(t+b)
$$

is strictly convex on $(-t, \infty)$ for any fixed $t>0$. The evident equalities

$$
\eta_{t}(0)=\eta_{t}(1)=0
$$

imply $\eta_{t}(b)<0$ for $b \in(0,1)$ and $\eta_{t}(b)>0$ if $b \in(1, \infty)$ or $b \in(-t, 0)$. This proves (a).

The computation of limit in (b) is standard. Differentiating $f$ we get $f^{\prime \prime}(t)=$ $\Psi^{\prime}(t)-\frac{1}{t}-\frac{1}{2 t^{2}}$, where $\Psi^{\prime}(t)=\frac{d^{2}}{d t^{2}} \ln \Gamma(t)=\sum_{n=0}^{\infty} \frac{1}{(t+n)^{2}}$. We have

$$
\begin{array}{r}
\Psi^{\prime}(t)=\frac{1}{2 t^{2}}+\frac{1}{2} \sum_{n=0}^{\infty}\left(\frac{1}{(t+n)^{2}}+\frac{1}{(t+n+1)^{2}}\right)>\frac{1}{2 t^{2}}+\sum_{n=0}^{\infty} \int_{n}^{n+1} \frac{d \tau}{(t+\tau)^{2}} \\
=\frac{1}{2 t^{2}}+\int_{0}^{\infty} \frac{d \tau}{(t+\tau)^{2}}=\frac{1}{t}+\frac{1}{2 t^{2}}
\end{array}
$$

where the inequality holds since the function $\frac{1}{t^{2}}$ is strictly convex. It follows that $f^{\prime \prime}(t)>0$ on $(0, \infty)$. Thus, (b) is true.

The function $f$ decreases since it is convex and has a finite limit at infinity. Therefore,

$$
f\left(\frac{1}{2}\right)=\ln \sqrt{\pi}>f(t)>\ln \sqrt{\frac{2 \pi}{e}}=\lim _{\tau \rightarrow \infty} f(\tau)
$$

for all $t$ in $\left(\frac{1}{2}, \infty\right)$. This proves (c).

Let $\mathfrak{h}_{p}$ denote the Lie algebra of the stable subgroup of $p \in M$ and $\pi_{p}$ be the orthogonal projection in $\mathcal{E}$ onto $T_{\bar{p}} \bar{M}=d_{p} \iota\left(T_{p} M\right)$.

Lemma 5. All functions $u \in \mathcal{S}$ are Lipschitz with the coefficient $\kappa=$ cs. Moreover, this coefficient is attained if and only if $u=\frac{1}{\kappa} \xi \phi_{p}$ for some $p \in M$ and $\xi \in \mathfrak{g}$ such that $\xi \perp \mathfrak{h}_{p},|\xi|=1$.

Proof. For any $u \in \mathcal{S}$ and $p \in M$ we have $u(p)=c\langle u, \bar{p}\rangle$. Since the mapping $p \rightarrow \bar{p}$ is a local metric homothety with the coefficient $s$ and the linear function $\ell_{u}(v)=\langle u, v\rangle$ is Lipschitz in $\mathcal{E}$ with the coefficient 1, the first assertion follows. Furthermore, the gradient of the restriction of $\ell_{u}$ onto $\bar{M}$ may be identified with $\pi_{p} u$. Hence, $\max _{q \in M}|\nabla u(q)|=\kappa$ if and only if $u \in T_{\bar{p}} \bar{M}$ for some $p \in M$. This happens if and only if $u$ is proportional to $\xi \phi_{p}$ for some $\xi \in \mathfrak{g}$ and $|u|=1$. A description of such $u$ is given in the statement of the lemma. 
Let $B(p, r)=\{q \in M: \rho(q, p)<r\}$ be the ball with respect to the Riemannian distance $\rho$ in $M$. Clearly, there exist $b>0$ and $r_{0}>0$, which depend only on the geometry of $M$, such that

$$
\mathfrak{h}^{m}(B(p, r))>b \varpi r^{m}
$$

for all $r \in\left(0, r_{0}\right)$.

Lemma 6. Let $u$ be a $\kappa$-Lipschitz function on $M, a \geq 1$, and $b, r_{0}$ be as above. Then

$$
\|u\|_{\infty} \leq b^{-\frac{1}{a}} r^{-\frac{m}{a}}\|u\|_{a}+\kappa r .
$$

for all $r \in\left(0, r_{0}\right)$.

Proof. We may assume that $\|u\|_{\infty}=\sup _{p \in M} u(p)$ replacing $u$ with $-u$ if necessary. According to the Chebyshev inequality, $\frac{1}{\varpi} \mathfrak{h}^{m}\left(U_{u}^{t}\right) \leq \frac{\|u\|_{a}^{a}}{t^{a}}$ for all $t>0$. Hence, if $p \in M$ and

$$
\frac{\|u\|_{a}^{a}}{t^{a}}<\frac{1}{\varpi} \mathfrak{h}^{m}(B(p, r)),
$$

then the ball $B(p, r)$ contains a point $q \notin U_{u}^{t}$. We have $\rho(p, q)<r$ and $u(q)<t$; it follows that $u(p)<t+\kappa r$. Moreover, $\|u\|_{\infty}<t+\kappa r$ since $t$ and $r$ are independent of $p$. If $\|u\|_{a}^{a} t^{-a}=b r^{m}$ and $r<r_{0}$, then (51) is true due to (49). This proves (50).

Theorem 4. Let $\mathcal{E}_{n}$ be a sequence of subspaces of $L^{2}(M)$ which satisfy $(\mathrm{E})$. Suppose $d_{n} \rightarrow \infty$ as $n \rightarrow \infty$. Then

$$
\begin{aligned}
& \lim _{n \rightarrow \infty} \mathrm{M}\left(\int_{M}|u(p)|^{a} d p\right)=\frac{2^{\frac{a}{2}}}{\sqrt{\pi}} \Gamma\left(\frac{a+1}{2}\right), a>-1, \\
& \limsup _{n \rightarrow \infty} \mathrm{M}\left(\|u\|_{a}\right) \leq \sqrt{2} \pi^{-\frac{1}{2 a}} \Gamma\left(\frac{a+1}{2}\right)^{\frac{1}{a}}<\sqrt{\frac{a+1}{e},} \quad a \geq 1 .
\end{aligned}
$$

Moreover, for any space $\mathcal{E}$ satisfying $(\mathrm{E})$

$$
\begin{gathered}
\mathrm{M}\left(\|u\|_{a}\right)<\sqrt{\frac{a+1}{e}}, \quad a \geq 2, \\
\mathrm{M}\left(\|u\|_{\infty}\right)<K(\sqrt{\ln \kappa}+1),
\end{gathered}
$$

where $\kappa=c s$ and $K>0$ is independent of $\mathcal{E}$.

Proof. The equality (52) follows from Theorem 3 and Lemma 4. (a), with $t=$ $\frac{d+1}{2}$ and $b=\frac{a}{2}$.

If $a>2$, then $\varphi_{b}(t)<1$ due to Lemma 4 (a); this proves the inequality

$$
\mathrm{M}\left(\|u\|_{a}^{a}\right)<\frac{2^{\frac{a}{2}}}{\sqrt{\pi}} \Gamma\left(\frac{a+1}{2}\right) .
$$


Set $t=\frac{a+1}{2}$. Then $t-\frac{1}{2}=\frac{a}{2}$. By Lemma 4 (a), for all $a>0$ we have

$$
\left(\frac{2}{e}\right)^{\frac{1}{2 a}} \sqrt{\frac{a+1}{e}}<\left(\frac{2^{\frac{a}{2}}}{\sqrt{\pi}} \Gamma\left(\frac{a+1}{2}\right)\right)^{\frac{1}{a}}<\sqrt{\frac{a+1}{e}} .
$$

Since $M\left(\|u\|_{2}\right)=1<\sqrt{\frac{3}{e}}$, we get (154). Moreover, (156) and (152) imply (153) for $a \geq 1$ :

$$
\limsup _{n \rightarrow \infty} \mathrm{M}\left(\|u\|_{a}\right) \leq \limsup _{n \rightarrow \infty} \mathrm{M}\left(\|u\|_{a}^{a}\right)^{\frac{1}{a}}=\left(\frac{2^{\frac{a}{2}}}{\sqrt{\pi}} \Gamma\left(\frac{a+1}{2}\right)\right)^{\frac{1}{a}}<\sqrt{\frac{a+1}{e}} .
$$

Due to Lemma 5, we may use Lemma 6 with $\kappa=c s$. Setting $r=\frac{1}{\kappa}$ and assuming $\kappa$ sufficiently large, we get

$$
\|u\|_{\infty} \leq b^{-\frac{1}{a}} \kappa^{\frac{m}{a}}\|u\|_{a}+1 .
$$

If $a=\ln k$, then $\kappa^{\frac{m}{a}}=e^{m}$ and $b^{-\frac{1}{a}} \leq \max \left\{1, b^{-1}\right\}$. Integrating over $\mathcal{S}$ and using (54), we get $\mathrm{M}\left(\|u\|_{\infty}\right) \leq K \sqrt{\ln \kappa}+1$ with some $K>0$.

We may assume $b$ arbitrary close to 1 making $r$ smaller if necessary. Thus, the inequality holds for any $K>e^{m-\frac{1}{2}}$ if $\kappa$ is sufficiently large.

\section{References}

[1] Bateman H., Erdélyi A., Higher transcendental functions, v. 2, MC Graw-Hill Book Company, New York, London, 1953.

[2] Bierstone E., Milman P., Semianalytic and subanalytic sets, IHES Publ. Math. 67 (1988), 5-42.

[3] Berard P., Volume des ensembles nodaux des fonctions propres du Laplacien. In Séminaire Bony-Sjöstrand-Meyer, Ecole Polytechnique, 19841985. Exposé $n^{\circ}$ XIV.

[4] Bloch A., Polya G., On the Zeros of Certain Algebraic Equations. Proc. London Math. Soc. 33, 102-114, 1932.

[5] Bogomolny E., Bohigas O., Leboeuf P., Quantum chaotic dynamics and random polynomials, J. Statist. Phys. 85 (1996), no. 5-6, 639-679.

[6] Bochnak J., Coste M., Roy M.-F., Real Algebraic Geometry, Ergebnisse der Mathematik und ihrer Grenzgebiete, 3, Folge A Modern Surveys in Mathmatics, 36, Springer-Verlag, Berlin, 1998.

[7] Courant R., Hilbert D., Methoden der Mathematischen Physik, Berlin, Verlag von Julius Springer, 1931. 
[8] Edelman A., Kostlan E., How many zeros of a random polynomial are real? Bull. Amer. Math. Soc. 32 (1995), 1-37.

[9] Federer H., Geometric Measure Theory, Springer, 1969.

[10] Gichev V.M., A note on common zeroes of Laplace-Beltrami eigenfunctions, Ann. of Global Anal. and Geom. 26 (2004), 201-208.

[11] Gichev V.M., Some remarks on spherical harmonics, St. Petersburg Math. J. 20 (2009), 553-567. (Original publication: Algebra i Analiz, tom 20 (2008), nomer 4 (Russian).)

[12] Granville A., Wigman I., The distribution of the zeros of random trigonometric polynomials, Amer. J. of Math., V. 133, No. 2, April 2011, pp. $295-357$.

[13] Heintze E., Ziller W., Isotropy irreducible spaces and s-representations, Diff. Geom. and Appl., V. 6 (1996), no. 2, P. 181-188.

[14] Jakobson D., Nadirashvili N., Toth J., Geometric properties of eigenfunctions. (English. Russian original.) Russ. Math. Surv. 56, No. 6, 1085-1105 (2001); translation from Usp. Mat. Nauk 56, No. 6, 67-88 (2001).

[15] Kac M., On the average number of real roots of a random algebraic equation, Bull. Amer. Math. Soc. 49, (1943). 314-320.

[16] Kahane J.-P., Some random series of fuctions, D.C. Heath and company, Lexington, Massachusetts, 1968.

[17] Krämer M., Eine Klassifikation bestimmter Untergruppen kompakter zusammenhiingender Liegruppen, Comm. Algebra, 3 (1975), 691-737.

[18] Ledoux M., The Concentration of Measure Phenomenon, Math. Surveys and Mono. 89, Amer. Math. Soc., Providence, RI, 2001.

[19] Littlewood J.E., Offord A.C., On the number of real roots of a random algebraic equation, J. London Math. Soc. vol. 13 (1938) pp. 288-295.

[20] Littlewood J.E., Offord A.C., On the number of real roots of a random algebraic equation II, Proc. Cambridge Philos. Soc. 35 (1939), 133-148.

[21] Manturov O.V., Homogeneous asymmetric Riemannian spaces with an irreducible group of motions, Dokl. Akad. Nauk SSSR, 141 (1961), 792795 .

[22] Manturov O.V., Riemannian spaces with orthogonal and symplectic groups of motions and an irreducible group of rotations, DoM. Akad. Nauk. SSSR, 141 (1961), 1034-1037.

[23] Manturov O.V., Homogeneous Riemannian manifolds with irreducible isotropy group, Trudy Sem. Vector. Tenzor. Anal., 13 (1966), 68-145. 
[24] Marinucci D., Wigman I., On the area of excursion sets of spherical Gaussian eigenfunctions, J. Math. Phys. 52, 093301 (2011); doi:10.1063/1.3624746 (21 pages).

[25] Marinucci D., Wigman I., The defect variance of random spherical harmonics, J. Phys. A, Math. Theor. 44, No. 35, Article ID 355206, 16 p. (2011); arXiv: physics. math-ph. 1103.0232.

[26] Milnor J.W., Morse Theory, Annals Math. Study 51, Princeton Univ. Press, 1963.

[27] Neuheisel J., The asymptotic distribution of nodal sets on spheres, Johns Hopkins Ph.D. thesis (2000).

[28] Oravecz, F., Rudnick, Z., and Wigman, I., The Leray measure of nodal sets for random eigenfunctions on the torus, Annales de linstitut Fourier, 58 no. 1 (2008), p. 299-335.

[29] Palais, R.S., Terng, C.-L.: Critical point theory and submanifold geometry. Lecture Notes in Mathematics 1353, Springer-Verlag, Berlin, New York, 1988.

[30] Paley R.E., Wiener N., and Zygmund Z., Notes on random functions, Math. Z. 37 (1932), 647-68.

[31] Qualls C., On the number of zeros of a stationary Gaussian random trigonometric polynomial, J. London Math. Soc. (2) 1970, 216-220.

[32] Rudnick Z., Wigman I., On the volume of nodal sets for eigenfunctions of the Laplacian on the torus, Ann. Henri Poincare 9 (2008), no. 1, 109-130.

[33] Salem R., Zygmund A., Some properties of trigonometric series whose terms have random signs, Acta Math. 91 (1954), 254-301.

[34] Shiffman B., Zelditch S., Random polynomials of high degree and Levy concentration of measure, Asian J. Math. 7 (2003), no. 4, 627646.

[35] Sogge C.D., Oscillatory integrals and spherical harmonics, Duke Math. J. 53 (1986), 43-65.

[36] Sogge C.D., Concerning the $L^{p}$ norm of spectral clusters for second-order elliptic operators on compact manifolds, J. Funct. Anal. 77 (1988), 123138.

[37] Sogge C.D., Zeldich S.,Concerning the $L^{4}$ norms of typical eigenfunctions on compact surfaces, arXiv:1011.0215 [math.AP].

[38] Wang M., Ziller W., On isotropy irreducible Riemannian manifolds, Acta Math., 166 (1991), 223-261. 
[39] Wigman I., On the distribution of the nodal sets of random spherical harmonics, J. Math. Phys. 50, -013521 (2009); doi:10.1063/1.3056589 (44 pages); arXiv:0805.2768v2 [math-ph].

[40] Wigman I., Fluctuations of the nodal length of random spherical harmonics, Comm. Math. Phys., 2010, V. 298, No. 3, P. 787-831. Fluctuations of the Nodal Length of Random Spherical Harmonics, erratum, arXiv: math. 0907.1648.

[41] Wolf J.A., The geometry and structure of isotropy irreducible homogeneous spaces, Acta Math., 120 (1968), 59-148; correction, Acta Math., 152 (1984), 141-142.

[42] Zelditch S., Local and global analysis of eigenfunctions on Riemannian manifolds. Ji, Lizhen (ed.) et al., Handbook of geometric analysis. No. 1, Somerville, MA: International Press; Beijing: Higher Education Press. Advanced Lectures in Mathematics (ALM) 7, 545-658 (2008). arXiv:0903.3420v1 [math.AP].

[43] Zelditch S., Eigenfunctions and nodal sets, arXiv:1205.2812 [math.SP].

[44] Zygmund A., On Fourier coefficients and transforms of two variables, Studia Math. 50 (1974), 189-201.

V.M. Gichev

Omsk Branch of Sobolev Institute of Mathematics

Pevtsova, 13, 644099, Omsk, Russia

gichev@ofim.oscsbras.ru 